\title{
Simplified laparoscopic technique for the treatment of long distal ureteral stenosis
}

\author{
Adam Golab, Marcin Slojewski, Andrzej Sikorski \\ Department and Clinic of Urology and Urological Oncology, Pomeranian Medical University, Szczecin, Poland
}

Videosurgery Miniinv 2013; 8 (4): 346-351

DOI: $10.5114 /$ wiitm.2011.35036

\begin{abstract}
Laparoscopic interventions with the application of vesico-psoas hitch and Boari flap methods for the reconstruction of pronounced ureter defects require considerable physical skill and competence. This case series aims to present our experience in the treatment of long, distal ureteral stenoses via simplified laparoscopic ureteroneocystostomy in 3 male patients in whom the stenoses ranged from $5 \mathrm{~cm}$ to $6 \mathrm{~cm}$. Prior to each laparoscopic intervention, intravenous urography and pyelography via a nephrostomy tube were performed. The mean operation time was $157 \mathrm{~min}$ (120-180 min), and blood loss averaged $33 \mathrm{ml}(20-50 \mathrm{ml})$. No intra-operative or post-operative complications were recorded. The postoperative hospital stay was $\leq 6$ days. Follow-up imaging examinations revealed normal kidney-urinary bladder urine flow in all cases. In conclusion, we consider that simplified ureteral replantation facilitates laparoscopic treatment of long, distal ureteral stenoses.
\end{abstract}

Key words: laparoscopy, ureter, replantation.

\section{Introduction}

Ureterocystoneostomy is the widely accepted treatment method for distal ureteral strictures [1]. Apart from simple anastomoses, the classical open method is useful for performing complex techniques such as vesico-psoas hitch, Boari flap, supplementing the ureteral defect with part of an intestine, or even renal autotransplantation [2]. For achieving comparable functional outcomes in the less invasive laparoscopic surgical method, the application of these techniques requires advanced skills and competence in reconstructive interventions, in particular, for intracavitary sutures [3]. However, gaining experience in reconstructive laparoscopy is fairly difficult due to the limited number of patients who qualify for such pro- cedures. Simplifying the laparoscopic technique may contribute to popularisation of this method in the treatment of ureteral stenoses.

To present our experience in the treatment of long, distal ureteral segment stenoses via the application of simplified laparoscopic ureteral replantation.

\section{Case report \\ Patients}

Three male patients underwent laparoscopic surgical correction of distal left ureteral stenosis with symptomatic hydronephrosis. The demographic and clinical characteristics of the patients are set out in Table I. In 2 of these patients, ureteral stenosis was 
Table I. Patient characteristics

\begin{tabular}{|cccccc|}
\hline Patient & $\begin{array}{c}\text { Age } \\
\text { [years] }\end{array}$ & BMI $^{*}$ & $\begin{array}{c}\text { Past abdominal } \\
\text { cavity surgeries }\end{array}$ & $\begin{array}{c}\text { Primary stage } \\
\text { of bladder carcinoma }\end{array}$ & $\begin{array}{c}\text { Lenght } \\
\text { of stenosis [cm] }\end{array}$ \\
\hline 1 & 41 & 30 & None & pTaNoMo G I & 6 \\
\hline 2 & 73 & 23 & Appendectomy & pT1NoMo G II & 6 \\
\hline 3 & 46 & 24 & Cholecystectomy & No cancer & 5 \\
\hline
\end{tabular}

${ }^{\star} B M 1-$ body mass index

a result of fibrosis following transurethral resection of non-invasive bladder carcinoma. In the remaining patient, cicatrisation occurred following ureteroscopic lithotripsy. The diagnosis of ureteral stenosis was confirmed by urography and pyelography, performed after implantation of the nephrostomy tube (Photo 1). The lengths of the stenoses were $6 \mathrm{~cm}, 6 \mathrm{~cm}$, and $5 \mathrm{~cm}$. The 2 patients with an oncological history underwent mapping biopsies of the bladder, which revealed no neoplastic process recurrence.
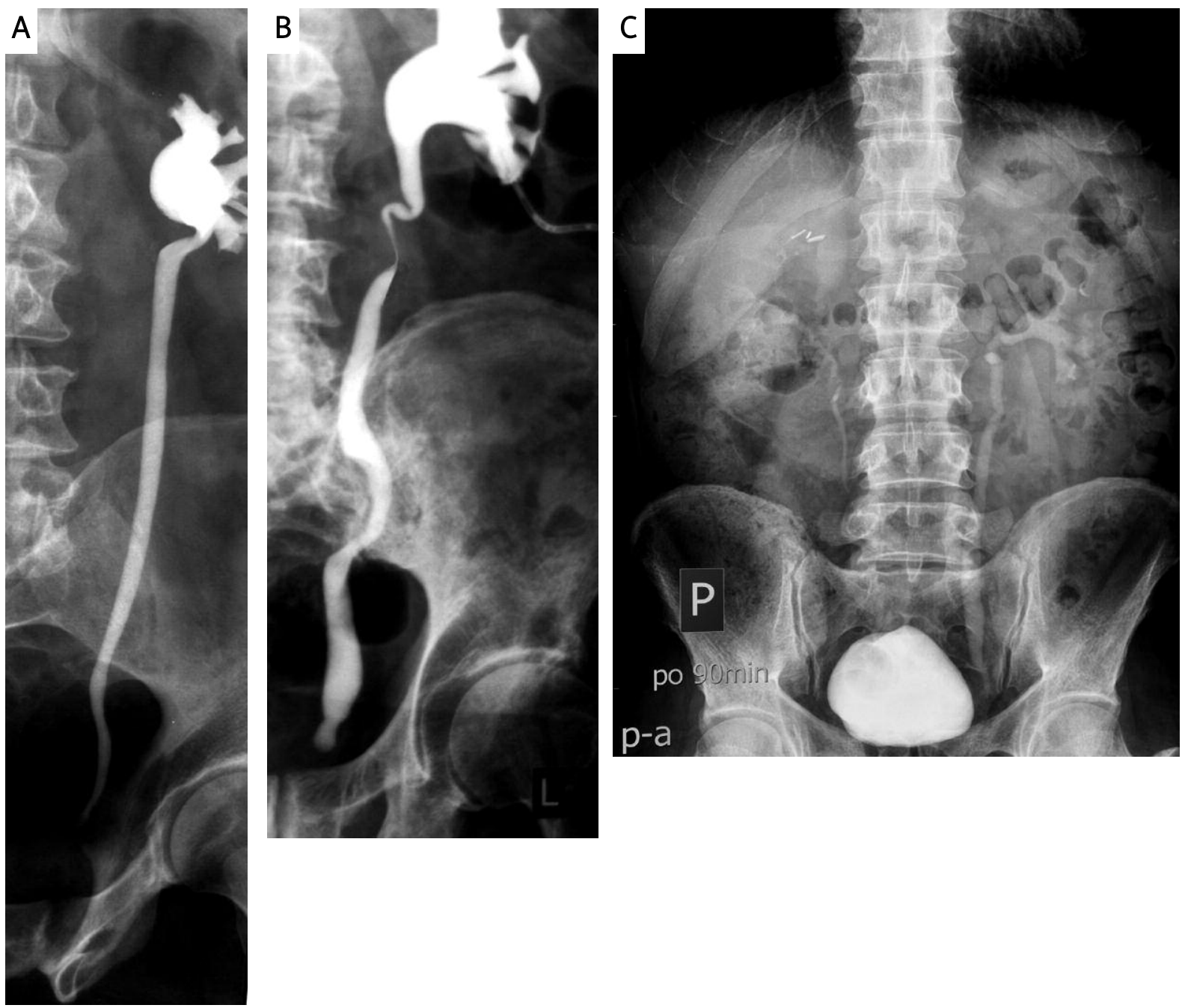

Photo 1. Pre-operative pyelography in patients 1 (A), 2 (B), and urography in patient 3 (C) 


\section{Surgical technique}

All operations were performed via the trans-peritoneal approach. Following the induction of general anaesthesia and intubation, we performed a cystoscopy. A Foley $18 \mathrm{~F}$ catheter was left in the bladder following the examination. The patient was placed in a Trendelenburg position, with the operation site elevated at a $45^{\circ}$ angle. A $10-\mathrm{mm}$ camera port was introduced near the umbilicus. Upon $\mathrm{CO}_{2}$ insufflation up to $12 \mathrm{~mm} \mathrm{Hg}, 3$ trocars were placed in the peritoneal cavity as follows: a 10-mm trocar in between the umbilicus and the pubic symphysis, and two 5-mm trocars in between the umbilicus and the anterior superior iliac spine, placed 4-5 cm apart. The parietal peritoneum was dissected along Toldt's line; the large intestine was freed and the iliac vessels near the ureter were exposed. Following ureter identification and its mobilisation to the level of the bladder, the stenosed portion was excised and sent for histopathological examination. A 2-cm long portion of the proximal end of the excised ureter was spatulated; then, a double-J $6 \mathrm{~F}$ catheter was introduced through one of the ports into the peritoneal cavity and ureteral lumen. Subsequently, the urinary bladder was filled with $250 \mathrm{ml}$ of saline solution, the covering peritoneum was incised, and the bladder was exposed by blunt dissection after immobilising the sides and the retropubic Retzius space. Filling the bladder with saline facilitated the identification of an optimal location for ureteral implantation as there was no tension or bends in the bladder surface. Next, the detrusor muscle was incised for $2 \mathrm{~cm}$ with a diathermic knife. The bladder mucosa was cut with scissors without the use of diathermy.
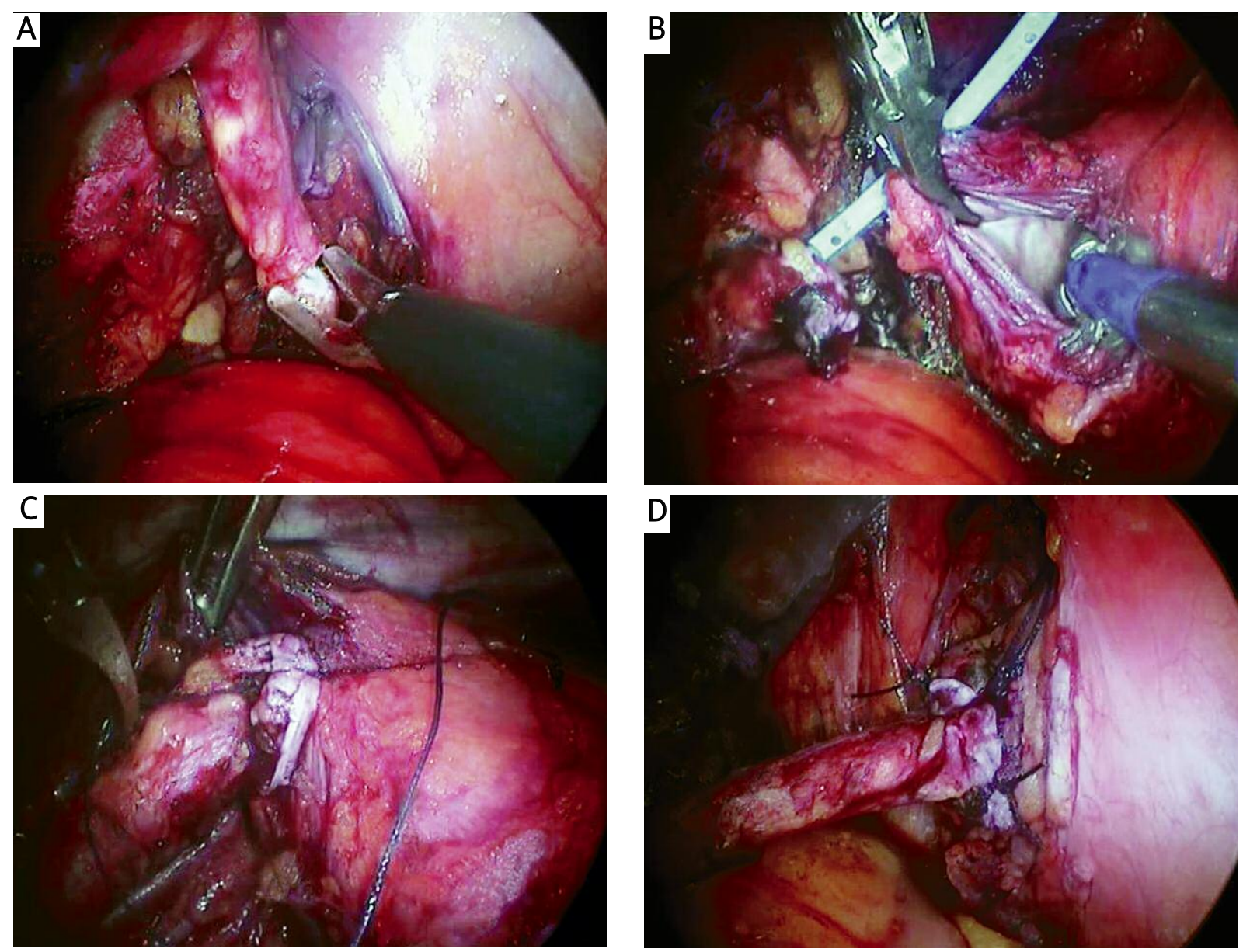

Photo 2. Laparoscopic replantation of the left ureter: A - excising the stenotic urethral segment; B - opening of the urinary bladder; $C$ - vesico-ureteral suture; D - final effect following the application of four vesico-ureteral sutures 
The Foley catheter was closed to prevent $\mathrm{CO}_{2}$ outflow from the peritoneal cavity. After opening the bladder, the free end of the double-J catheter was placed into the bladder and a tension-free ureteral reimplantation was performed. Single, absorbable Vicryl 3-0 sutures were applied to the whole ureteral and bladder wall, and intracavitary binding was performed. The procedure was concluded with an evaluation of the tightness of the bladder by filling it with $200 \mathrm{ml}$ of saline solution, drainage, and skin suture (Photo 2).

\section{Results}

Basic data regarding the course of operations are presented in Table II. The post-operative hospital stay was 5 days in 2 of the patients, and 6 days in 1 case. The nephrostomy tube was removed on post-operative day 2 ; the peritoneal drain, on post-operative day 3; Foley's catheter, at post-operative week 2; and the double-J catheter, after 4 weeks post-operatively. The efficacy of the treatment was assessed based on clinical examination, ultrasound examination, and intravenous urography performed 1 or 2 days after the removal of the ureteral double-J catheter. In each case, the urinary kidney-bladder flow was appropriate (Photo 3). Cystoscopy performed after 3 and 12 months post-operatively revealed the natural appearance of the bladder after surgery and the implanted ureteral ostium. No recurrent urinary bladder carcinoma was noted.

\section{Discussion}

Ureteral replantation is a procedure performed most often in children with vesico-ureteral reflux. Indications for such surgery in adults are much less common, being usually required for iatrogenic ureteral damage, with stenosis or fistula. The open surgical technique is commonly used for such ureteral repair. Laparoscopic ureter replantation was first reported by Reddy and Evans in 1994 [4]. Subsequent related publications have presented short series of patients, the largest of which $(n=45)$ was presented by Seideman et al. [1]. Previous papers have highlighted the low invasiveness of laparoscopic treatment in comparison with open surgery, comparable functional outcomes, and the low risk of subsequent complications along with the necessity of wide experience with the laparoscopic technique [1-5]. The last element of surgical skill significantly affects the small size of the cohorts presented in the studies. Performing complex anastomoses with the use of the Boari flap and vesico-psoas hitch is reportedly most difficult, with an average operative time of $247 \mathrm{~min}$, as reported by Gözen et al. [5]. Operations carried out without the employment of the above techniques were significantly shorter as they lasted, on average, 156 min [5]. The Boari flap facilitates tension-free anastomosis in the case of larger ureteral defects [6]. Despite the presence of large ureteral deficiencies in our present patients, we avoided the Boari flap method, each time performing high dissection towards the hilum of the kidney, which served the purpose of creating an adequate anastomosis. Another important aspect was the careful mobilisation of the bladder walls. We did not record any elevated tension in the anastomosis, fibrosis with restenosis, or lack of tightness in our cases, all of which are reported to occur frequently [5].

Creating an anti-reflux mechanism is one of the components of the ureteral replantation procedure. The technical aspects of such laparoscopic surgery have been well described by Rassweiler et al. [2] and Simmons et al. [7]. The most commonly used method is the Lich-Gregoir technique, which requires some experience in laparoscopic treatment, similarly to the previously mentioned procedures. However, a significant ureteral defect prevents the application of antireflux methods. Therefore, we performed simple ureteroneocystostomy in our patients, all of whom had significant ureteral defects. Potentially, this tech-

Table II. Perioperative data

\begin{tabular}{|ccccc|}
\hline Patient & $\begin{array}{c}\text { Operation } \\
\text { duration [min] }\end{array}$ & $\begin{array}{c}\text { Blood } \\
\text { loss [ml] }\end{array}$ & $\begin{array}{c}\text { Number of ureteroneo- } \\
\text { cystostomy sutures }\end{array}$ & $\begin{array}{c}\text { Intra- and postoperative } \\
\text { complications }\end{array}$ \\
\hline 1 & 170 & 30 & 6 & None recorded \\
\hline 2 & 120 & 20 & 4 & None recorded \\
\hline 3 & 180 & 50 & 6 & None recorded \\
\hline
\end{tabular}



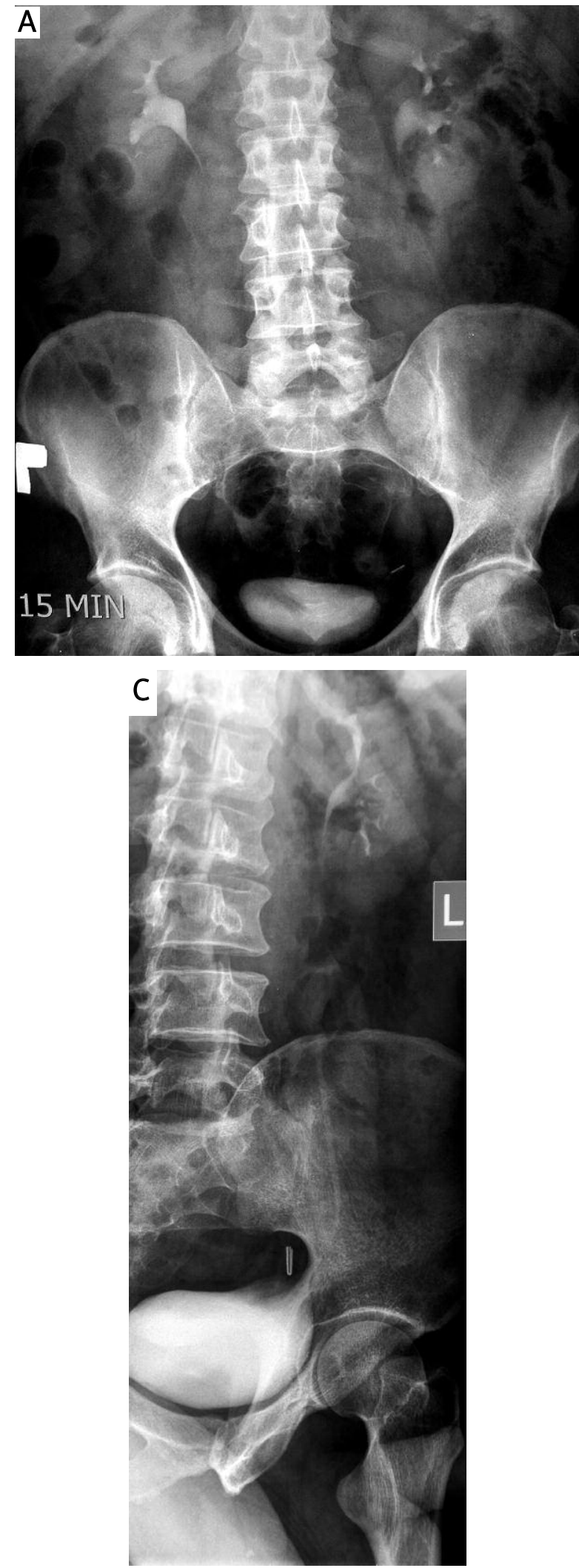

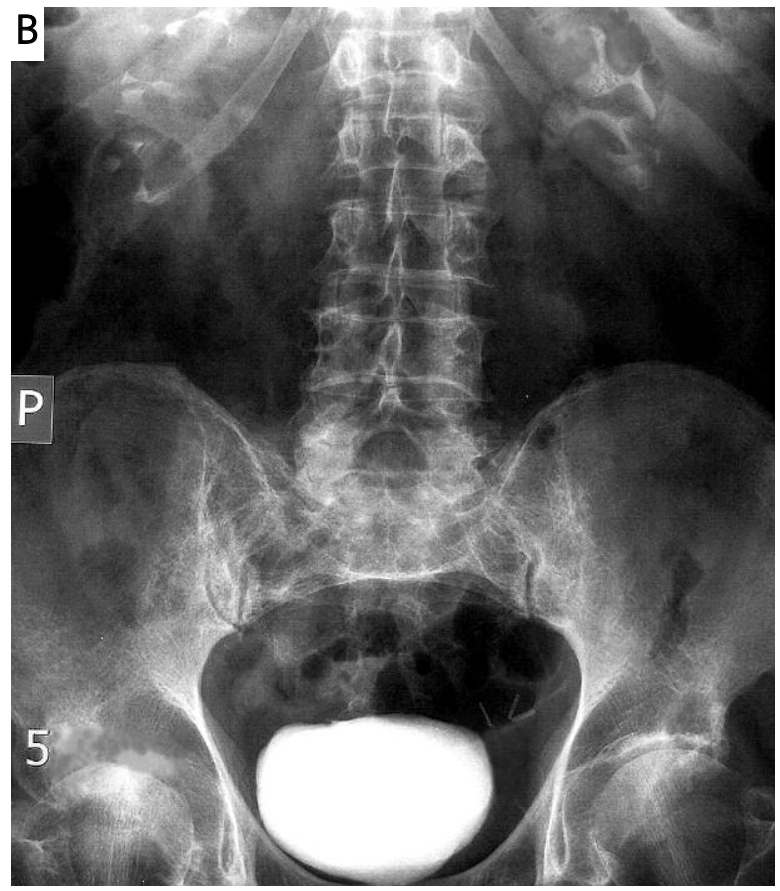

nique does increase the risk of reflux; however, in practice, it is considered that in adults, a reflux unaccompanied by recurring urinary tract infections has an insignificant effect on renal function [8, 9]. We observed no inflammatory complications or worsening of the renal function in our male subjects at 12-month follow-up.

In conclusion, based on our experience, we consider that the implementation of simple laparoscopic manoeuvres, which require only the basic knowledge of laparoscopic intracavitary suture techniques, can be successfully employed for reconstructive surgery of the distal ureter, even in patients with long stenoses. The application of robotic surgery in this technique, with its commonly recognised benefits, such as 3D imaging and great freedom of movement, will most certainly enhance the rapid development of this method [10].

Simplified ureteral replantation, i.e. ureteroneocystostomy, facilitates the laparoscopic treatment of long, distal ureteral stenoses.

Photo 3. Intravenous post-operative urography in patients 1 (A), 2 (B), and 3 (C) 


\section{References}

1. Seideman CA, Huckabay C, Smith KD, et al. Laparoscopic ureteral reimplantation: technique and outcomes. J Urol 2009; 181 1742-6.

2. Rassweiler JJ, Gözen AS, Erdogru T, et al. Ureteral reimplantation for management of ureteral strictures: a retrospective comparison of laparoscopic and open techniques. Eur Urol 2007; 51: 512-22.

3. Gao J, Dong J, Xu A, et al. A simplified technique for laparoscopic ureteroneocystostomy without ureteral nipple or submucosal tunneling. J Endourol 2007; 21: 1505-8.

4. Reddy PK, Evans RM. Laparoscopic ureteroneocystostomy. J Urol 1994; 152: 2057-9.

5. Gözen AS, Cresswell J, Canda AE, et al. Laparoscopic ureteral reimplantation: prospective evaluation of medium-term results and current developments. World J Urol 2010; 28: 221-6.

6. Rassweiler J, Pini G, Gözen AS, et al. Role of laparoscopy in reconstructive surgery. Curr Opin Urol 2010; 20: 471-82.

7. Simmons MN, Gill IS, Fergany AF, et al. Technical modifications to laparoscopic Boari flap. Urology 2007; 69: 175-80.

8. Symons S, Kurien A, Desai M. Laparoscopic ureteral reimplantation: a single center experience and literature review. J Endourol 2009; 23: 269-74.

9. Fugita OE, Dinlenc C, Kavoussi L. The laparoscopic Boari flap. J Urol 2001; 166: 51-3.

10. Phillips EA, Wang DS. Current status of robot-assisted laparoscopic ureteral reimplantation and reconstruction. Curr Urol Rep 2012; 13: 190-4.

Received: 4.02.2013, accepted: 25.03.2013. 